\title{
Standard spatial graph
}

Dedicated to Professor Haruo Suzuki on his 60th birthday

\author{
Kazuaki KoBAYASHI
}

(Received February 12, 1991, Revised September 6, 1991)

\section{$\S 0$. Introduction}

In this article we consider the graph as a topological space or a $C W$ complex and define some kinds of standard embeddings of the graphs into the 3-dim. Euclidean space $R^{3}$ or the 3 -sphere $S^{3}$ and discuss the property. For example, for a knot or a link we can define the standard embedding the trivial knot or the trivial link. And although, by Fox's Theorem, for any finite graph $G$ there is a spatial graph $\widetilde{G}$ of $G$ such that the complementary space of the interior of a regular neighborhood of $\widetilde{G}$ is homeomorphic to a handlebody with genus equal to the $\operatorname{rank} H_{1}(G: Z)$, this spatial graph is not suitable to the "standard" spatial graph in general (see Figure 6). Furthermore the image of any embedding of the complete graph with $n$ vertices, $\widetilde{K}_{n}$, contains a non-trivial link for $n \geqq 6$ and contains a non-trivial knot for $n \geqq 7$ ([2]). So if we adopt as the definition of the standard embedding a spatial graph (i. e. the embedded image of the graph) which does not contain a non-trivial knot or link, the definition can not apply to all finite graph. Although there are concepts of minimal genus, maximal genus and thickness in the graph theory $([1])$, [11]]), these concepts do not satisfy the properties which should be satisfied by the " standard" embedding from view point of the knot theory. That is, the graph theoretical properties of the above are weak for the "standard" embedding from the knot theory. For example, although two spatial graphs of the complete graph $K_{5}$ of Figure 4 and 5 are both on the torus but the first one does not contain any non-trivial knot and the second one contains a trefoil knot.

From the knot theory, the properties which should be satisfied by the "standard embedding" (or the "standard spatial graph") are the following ;

Let $G$ be a finite graph and $\widetilde{G}$ be a "standard" spatial graph of $G$. Then 
(I) $\pi_{1}\left(R^{3}-\widetilde{G}\right)$ is the free group of rank equal to $\operatorname{rank} H_{1}(G: Z$ ) (see the following Remark),

(II) any graph $G$ has a "standard" spatial graph and

(III) two "standard" spatial graphs $\widetilde{G}_{1}$ and $\widetilde{G}_{2}$ of a graph $G$ are equivalent in some sense.

REMARK. For a compact orientable 3-manifold with boundary, $M$, it is homeomorphic to the handlebody if and only if $\pi_{1}\left(M^{3}\right)$ is free group ([3]).

For example if $G$ is a cycle graph (=homeomorphic to a circle), a disjoint union of cycle graphs (=homeomorphic to a disjoint union of circles) or a planar graph, the "standard" graph of those one are the trivial knot, the trivial link or the plane graph.

That is,

(1) a knot $K$ is trivial if and only if $\pi_{1}\left(S^{3}-K\right) \cong \boldsymbol{Z}$ ([6]) (2) a link $L$ is trivial if and only if $\pi_{1}\left(S^{3}-L\right) \cong \underbrace{Z_{*} \boldsymbol{Z} * \ldots * \boldsymbol{Z}}_{n}$ where $n$ is
the number of components of $L$, and

(3) for a planar graph $G$, its graph $\widetilde{G}$ is a plane graph if and only if for any spatial subgraph $\widetilde{H}$ of $\widetilde{G}, \pi_{1}\left(S^{3}-\widetilde{H}\right)$ is free $([7])$.

And these "standard" spatial graph of a graph are ambient isotopic each other by the ambient isotopy of $S^{3}$. So these "standard" spatial graphs satisfy the above conditions (I) -(III) where "some sence" in (III) means "ambient isotopy" in these cases.

REMARK. We can not adopt the necessary condition of the above example (3) as a condition of "standard embedding" because any embedding (or any spatial gragh) of the complete graph with $n$ vertices $(n \geqq 6)$ contains a non-trivial link and contains a non-trivial knot for $n \geqq 7$ ([2]). But any spatial subgraph with some restrictions of the "standard" spatial graph has a similar property (Corollary 2 to Proposition 9).

REMARK. We think that the ambient isotopy is too restrictive as "some sence" in (III). For this, see Remark after Proposition 13.

Throughout this paper, we work in the piecewise linear $(=\mathrm{PL})$ category or differential category.

In $\S 1$ we consider the spatial graph from knot and link theory and introduce concepts "locally unknotted, globally unknotted (unlinked)" and discuss the properties. Although "globally unknottedness" is too restricted as the definition of the "standard" spatial graph, it is convenient to use results of the knot theory. Local unknottedness gives some 
restriction on spatial graphs. But there is evident difference between "local unknottedness" and "global unknottedness" (example 2). And "local unknottedness" is something weak by examples 2 and 3 . So next we difine "locally unknotted with respect to a well situated base and with respect to a triangle base" which are more restricted concepts than simple "locally unknotted". These are useful for the complete graph. In $\$ \mathbf{2}$ we shall introduce "book presentation" and define the book presentation with minimum sheets which is probably a proper concept as the "standard" embedding of pseudo Hamilton graphs (see $\S 2$ and Definition 12).

I want to acknowledge my debt to the many people, colleagues in our seminar at Tokyo Woman's Christian University, especially Shin'ichi Suzuki (Waseda Univ.) who found some gaps and corrected many errors and graduate students T. Endo, K. Ikeda, A. Kaneko and C. Toda who determined the minimum number of sheets of a B.P.H. $\Delta$ of complete graphs $K_{n}$ (Proposition 11. (2)). And I also want to express my appreciation Korea Advanced Institute of Science and Technology, especially Gyo Taek Jin who invited me KAIST and gave me an opportunity to talk about this subject.

\section{§ 1. Local unknottedness and global unknottedness}

Through this paper we assume that the graph is finite and does not have vertices with degree 1 or 2 if otherwise stated. And as we consider the "standard" spatial graph, we may restrict the graph to be simple without cut edges. For the graph $G$ let $\gamma=\operatorname{rank} H_{1}(G: Z)$. We only use a base $\left\{x_{1}, \ldots x_{\gamma}\right\}$ for $H_{1}(G: Z)$ satisfying the condition that any element $x_{i}$ can be represented by a simple closed curve $C_{i}$. Let $f: G \longrightarrow R^{3}$ (or $S^{3}$ ) be an edgewise differentiable embedding and $\widetilde{G}=f(G)$. We call $\widetilde{G}$ a spatial graph of $G$.

DEFINITION 1. A spatial graph $\widetilde{G}$ is locally unknotted if there are a base $\left\{x_{1}, \ldots, \mathrm{x}_{\gamma}\right\}$ of $H_{1}(G: Z)$ and a map $\psi: \bigcup_{i=1}^{\gamma} D_{i}{ }^{2} \longrightarrow S^{3}$ such that

1) $\phi\left(\partial D_{i}^{2}\right)=\widetilde{C}_{i}$ where $\widetilde{C}_{i}$ is a representation curve of $x_{i}$ in $\widetilde{G}$.

2) $\phi\left(\bigcup_{i=1}^{\gamma} \partial D_{i}^{2}\right)=\widetilde{G}$

3) $\psi \mid D_{i}{ }^{2}=$ embedding for $i=1,2, \ldots, \gamma$

4) $\psi\left(\operatorname{Int}_{i}{ }^{2}\right) \cap \psi\left(\operatorname{Int} D_{j}^{2}\right)=\phi$ for $i \neq j$

5) $\phi\left(D_{i}^{2}\right) \cap \widetilde{G}=\psi\left(\partial D_{i}^{2}\right) \cap \widetilde{G}=\widetilde{C}_{i}$

Otherwise we call also $\widetilde{G}$ locally knotted.

DEFINITION 2. A spatial graph $\widetilde{G}$ is locally unknotted with respect to 
$a$ base $\left(x_{1}, \ldots, x_{\gamma}\right\}$ if for the distinguished base $\left\{x_{1}, \ldots, x_{r}\right\}$ there is a map $\psi: \bigcup_{i=1}^{\gamma} D_{i}{ }^{2} \longrightarrow S^{3}$ satisfying (1)-(5) of Definition 1 .

DEFINITION 3. We say that a spatial graph $\widetilde{G}$ is globally unknotted if, any simple closed curve $\widetilde{C}$ on $\widetilde{G}$ is the trivial knot. Otherwise we say also globally knotted.

DEFINITION 4. A spatial graph $\widetilde{G}$ is globally unlinked if for any set of disjoint simple closed curves, $\left\{\widetilde{C}_{i}\right\}, i=1,2, \ldots$, $\mu$ on $\widetilde{G}, \widetilde{C}_{1} \cup \ldots \cup \widetilde{C}_{\mu}$ is the trivial link.

Otherwise we say also globally linked.

PROPOSITION 1. Let $\widetilde{G}$ be a spatial graph. If for any spatial subgraph $\widetilde{H}$ of $\widetilde{G} \pi_{1}\left(S^{3}-\widetilde{H}\right)$ is free then $\widetilde{G}$ is globally unknotted and globally unliked.

PROOF. If $\widetilde{G}$ contains a non-trivial knot or non trivial link $\widetilde{C}$, $\pi_{1}\left(S^{3}-\widetilde{C}\right)$ is not free.

The converse of the above Proposition 1 does not holds. Figure 0. (S. Suzuki [10]) is a spatial graph of $K_{4}$ which is globally unknotted and globally unlinked. But $\pi_{1}\left(S^{3}-\widetilde{K}_{4}\right)$ is not free.

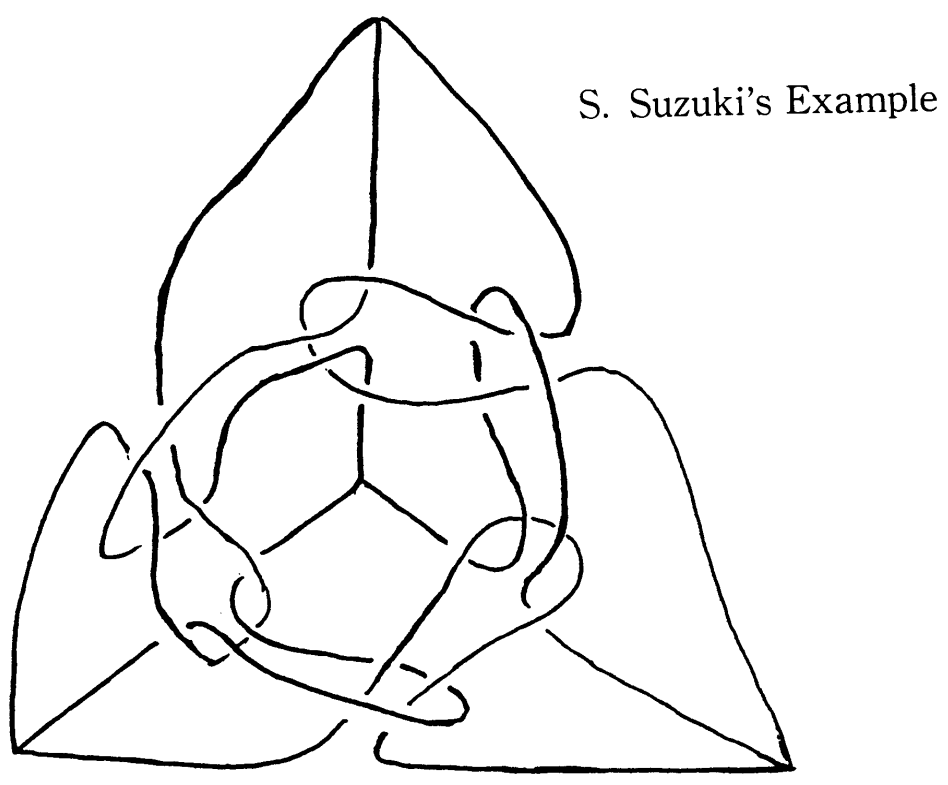

Fig. 0. 
DEFINITION 5. ([5], [9]) If for any spatial graph $\widetilde{G}$ of $G, \widetilde{G}$ is globally knotted, then we define $G$ to be self-knotted. And if for any spatial graph $\widetilde{G}$ of $G, \widetilde{G}$ is globally linked, then we call $G$ to be self-linked. According to ([2]), the complete graph with $n$-vertices, $K_{n}$, is self-linked provided $n \geqq 6$ and self knotted for $n \geqq 7$.

So the following holds.

PROPOSITION 2. If $G$ is a self-knotted graph, any spatial graph of $G$ is not globally unknotted. Similary if $G$ is a self-linked graph, any spatial graph of $G$ is not globally unlinked.

PROPOSITION 3. If a spatial graph $\widetilde{G}$ is contained in the 2-sphere $S^{2}$, then $\widetilde{G}$ is locally unknotted, globally unknotted and globally unlinked.

REMARK. The converse of Proposition 3 does not hold. Since the complete graph with 5 vertices, $K_{5}$, is not self-knotted and not self-linked, there is a spatial graph $\widetilde{K}_{5}$ which contains only trivial knots. But $K_{5}$ is not planer so $\widetilde{K}_{5}$ is not contained in $S^{2}$.

PROPOSITION 4. If a spatial graph $\widetilde{G}$ is locally unknotted then $\pi_{1}\left(S^{3}\right.$ $-\widetilde{G})$ is free.

PrOOF. Since $\widetilde{G}$ is locally unknotted, there are a base of $H_{1}(G: Z)$ and a map $\phi: \bigcup_{i=1}^{\gamma} D_{i}{ }^{2} \longrightarrow S^{3}$ satisfying the conditions of Definition 1. Put $P=\widetilde{G} \cup \psi\left(\bigcup_{i=1}^{\gamma} D_{i}{ }^{2}\right)$, then $P$ is a connected 2-dim. complex with $H_{1}(P: Z)=$ $\{0\}$. If $U(P)$ is a regular neighborhood of $P, U(P)$ is a bounded 3-manifold with homotopy type $P$. By the Alexander's duality theorem, $H_{1}\left(S^{3}\right.$ $-U(P)) \cong H_{1}(U(P)) \cong\{0\}$ and $H_{2}\left(S^{3}-U(P)\right) \cong \widetilde{H}_{0}(U(P)) \cong\{0\}$. Hence $S^{3}$ $-U(P)$ is acyclic and so $\partial U(P)$ is a 2 -sphere. By the Schoenflies theorem, $S^{3}-U(P)$ is a 3 -ball. So $S^{3}-\widetilde{G}$ is a 3 -ball with 1 -cells dual to 2 -cells $\bigcup_{i=1}^{\gamma} \psi\left(D_{i}^{2}\right)$ respectively and hence $S^{3}-\widetilde{G}$ is a handlebody. Therefore $\pi_{1}\left(S^{3}-\widetilde{G}\right)$ is free.

We denote $E(G)$ a set of edges of a graph $G$ and $|E(G)|$ a number of elements of the set $E(G)$.

LEMMA 1. Let $G$ be a connected graph and $T_{G}$ be a maximal tree of $G$. If $\gamma=\operatorname{rank} H_{1}(G: Z)$ then $\gamma=|E(G)|-\left|E\left(T_{G}\right)\right|$.

PROOF. Since $G$ is connected, by Euler's formula

$1-\gamma=|V(G)|-|E(G)|$. Since $T_{G}$ is a maximal tree of $G,\left|V\left(T_{G}\right)\right|=$ 
$|V(G)|$ and $\left|E\left(T_{G}\right)\right|=\left|V\left(T_{G}\right)\right|-1$. So $|V(G)|=\left|E\left(T_{G}\right)\right|+1$ and $1-\gamma=$ $\left|E\left(T_{G}\right)\right|+1-|E(G)|$. Hence $\gamma=|E(G)|-\left|E\left(T_{\mathrm{G}}\right)\right|$.

The following lemma is well known. So we omit its proof.

LEMMA 2. Let $G$ be a finite graph and $\gamma=\operatorname{rank} H_{1}(G: Z)$. For a set of simple closed curves on $G, \Gamma=\left\{C_{1}, C_{2}, \ldots, C_{r}\right\}$, we construct a 2-comlexes $P=G \cup_{f} \bigcup_{i=1}^{\gamma} D_{i}{ }^{2}$ where $f: \bigcup_{i=1}^{\gamma} \partial D_{i}{ }^{2} \longrightarrow G$ is a map so that $\left.f\right|_{\partial D_{i}^{2}}(i=1,2, \ldots, \gamma)$ is an embedding and $f\left(\partial D_{i}{ }^{2}\right)=C_{i}$. Then $\Gamma$ is a set of representation curves of $a$ base of $H_{1}(G: Z)$ if and if $P$ does not contain an image of an immerision of an orintable closed surfuce.

COROLLARY. Let $G$ be a finite graph and $\gamma=\operatorname{rank} \mathrm{H}_{1}(G: Z)$. If for $a$ set of simple closed curves on $G, \Gamma=\left\{C_{1}, \ldots, C_{\gamma}\right\}$, there is a maximal tree $T_{G}$ of $G$ satisfying the following condition $(*)$, then $\Gamma$ is in fact a set of representation curves of a base of $H_{1}(G: Z)$.

(*) Let $\Lambda$ be a $E(G)-E\left(T_{\mathrm{G}}\right)=\left\{e_{1}, e_{2}, \ldots, e_{r}\right\}$. For any element $e_{i} \in \Lambda$, there is only one $C_{j}$ in $\Gamma$ with $e_{i} \in E\left(C_{j}\right)$.

THEOREM 1. Any comlete graph has a locally unknotted spatial graph.

PROOF. Since $\left|V\left(K_{n}\right)\right|=n$ and $\left|E\left(K_{n}\right)\right|=n(n-1) / 2$, by Euler's formula $\gamma=(n-1) \times(n-2) / 2$ where $\gamma=\operatorname{rank} H_{1}\left(K_{n}: Z\right)$. Let $V\left(K_{n}\right)=\left\{v_{1}, \ldots, v_{n}\right\}$ and set $v_{k}=e^{2 \pi i(k-1) / n} \in C \quad k=1,2, \ldots, n$ where $S^{1}=\left\{e^{i \mathrm{e}} \mid 0 \leqq \theta \leqq 2 \pi\right\}$.

First join the pairs of vertices $v_{1} \& v_{2} ; v_{2} \& v_{3} ; \ldots ; v_{n-1} \& v_{n} ; v_{n} \&$ $v_{1}$, then $n$ edges were made. Second join the pairs of vertices $v_{1} \& v_{3} ; v_{1}$ $\& v_{4} ; \ldots ; v_{1} \& v_{n-1}$ then $(n-3)$ edges were made. And $(n-2)$ triangles $\Delta v_{1} v_{2} v_{3}, \Delta v_{1} v_{3} v_{4}, \ldots, \Delta v_{1} v_{n-1} v_{n}$ bound 2-cells respectively which do not intersect at their interiors. Third join the pairs of vertices $v_{2} \& v_{4} ; v_{2} \&$ $v_{5} ; v_{2} \& v_{6} ; \ldots ; v_{2} \& v_{n}$ which pass under the edges made by the second step then $(n-3)$-edges were made and $n$-3 triangles $\Delta v_{2} v_{3} v_{4}, \Delta v_{2} v_{4} v_{5}, \ldots$, $\Delta v_{2} v_{n-1} v_{n}$ bound 2-cells respectively such that these constructed 2-cells in the second and third steps do not intersect at their interiors. Fourth join the pairs of vertices $v_{3} \& v_{5} ; v_{3} \& v_{6} ; \ldots ; v_{3} \& v_{n}$ which pass under the edges made by the second and third steps then $n-4$ edges were made and $n$-4 triangles $\Delta v_{3} v_{4} v_{5}, \Delta v_{3} v_{5} v_{6}, \ldots, \Delta v_{3} v_{n-1} v_{n}$ bound 2-cells respectively such that these constructed 2-cells in the second, third and fourth steps do not intersect at their interiors. And so on. Finally join $v_{n-2}$ and $v_{n}$ whose edge is undermost among all edges constructed in the above steps. And a triangle $\Delta v_{n-2} v_{n-1} v_{n}$ bound a 2-cell which does not intersect the 
other 2-cells constructed by the above steps at their interiors (Fig. 1). The total number of constructed edges by the above is $n+(n-3)+((n$ $-2)(n-3) / 2)=n(n-1) / 2$ and the total number of interior disjoint 2-cells of the above is $(n-1)(n-2) / 2$ of which boundaries are a set of representation curves of a base of $H_{1}\left(K_{n}: Z\right)$ by lemma 2 .

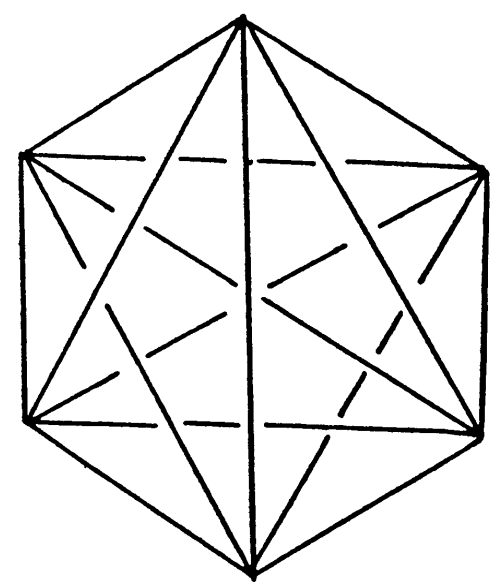

Fig. 1.

By the following examples, it is clear that the definition of locally unknottedness is something weak for us (i.e. as a "standard" spatial graph).

EXAMPLES 1. Locally unknotted complete graph $K_{n}$ for $n=3,4,5$. $n=3$. locally unknotted $\leftarrow \rightarrow$ globally unknotted

$$
\leftarrow \rightarrow \text { trivial knot as a knot }
$$

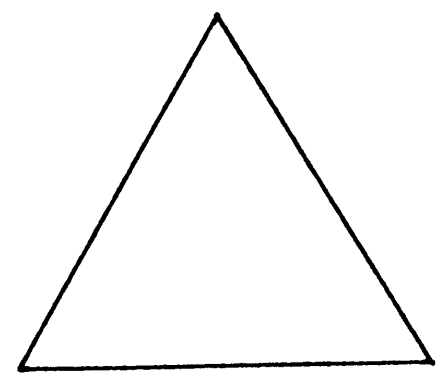

Fig. 2-1

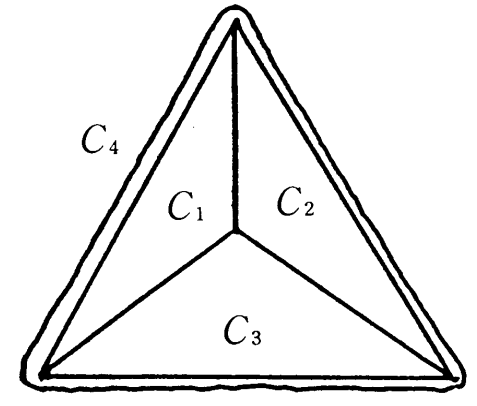

Fig. 2-2 
$n=4 . \quad \gamma=\operatorname{rank} H_{1}\left(K_{4}: Z\right)=3 . \quad$ Let $H_{1}\left(K_{4}: Z\right)=\left\langle x_{1}, x_{2}, x_{3} \mid\left[x_{i}, x_{j}\right]\right\rangle=B_{1}$

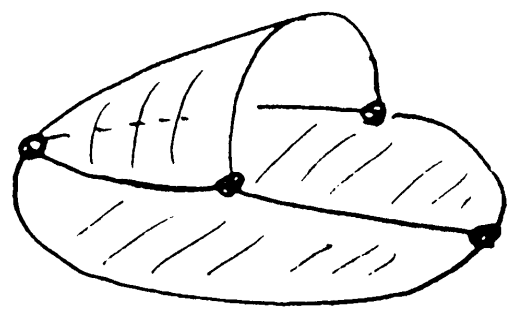
where $x_{1}=\left\langle C_{1}\right\rangle, x_{2}=\left\langle C_{2}\right\rangle$ and $x_{3}=\left\langle C_{3}\right\rangle$ and let $B_{2}=\left\langle x_{1}^{\prime}, x_{2}^{\prime}, x_{3}^{\prime} \mid\left[x_{i}^{\prime}, x_{j}^{\prime}\right]\right\rangle$ where $x_{1}^{\prime}=\left\langle C_{1} \# C_{2}\right\rangle$, $x_{2}{ }^{\prime}=\left\langle C_{3}\right\rangle$ and $x_{3}{ }^{\prime}=\left\langle C_{1}\right\rangle$. By the symmetry of $K_{4}$, there is only two way of the choice of the base of $H_{1}\left(K_{4}: Z\right)$ as above. If each base element of $B_{1}$ bounds a 2-cell, $\phi_{1}\left(D_{i}^{2}\right)$ satisfying the conditions of Definition 1, then $\widetilde{K}_{4} \cup$ $\psi_{1}\left(\bigcup_{i=1}^{3} D_{i}{ }^{2}\right) \cong D^{2}$ (a 2-cell). And if each bace element of $B_{2}$ bounds a 2-cell $\psi_{2}\left(D_{i}{ }^{2}\right)$ satisfying the conditions of Definition 1, then $\widetilde{K}_{4} \cup \psi_{2}\left(\bigcup_{i=1}^{3} D_{i}{ }^{2}\right) 2$-disk with a pocket (Figure 3). So for the first case with respect to the base $B_{1}$, $\widetilde{K}_{4}$ is globally unknotted. And the second case for $B_{2}$ is also globally unknotted by checking all 43 -cycles and 34 -cycles. Hence if $\widetilde{K}_{4}$ is locally unknotted, it is globally unknotted. And a locally unknotted $\widetilde{K}_{4}$ is unique up to ambient isotopy. But the converse is false. The spatial graph $\widetilde{K}_{4}$ of Figure 0 is globally unknotted but locally knotted.

For $n=5$, the complete graph with 5 vertices, $K_{5}$, has a spatial graph $\widetilde{K}_{5}$ which is locally unknotted but globally knotted (see Figure 5).

EXAMPLE 2. The spatial graph, $\widetilde{K}_{5}$, of Figure 4 is locally unknotted, globally unknotted, and $\pi_{1}\left(S^{3}-\widetilde{K}_{5}\right)$ is the free group of rank $6\left(=\operatorname{rank} H_{1}\right.$ $\left(K_{5}: Z\right)$ ).

The spatial graph, $\widetilde{K}_{5}$, of Figure 5 is locally unknotted, globally knotted and $\pi_{1}\left(S^{3}-\widetilde{K}_{5}\right)$ is the free group of rank 6 .

Although a multi-edge graph $\theta$ is not simple, the spatial graph, $\tilde{\theta}$, of Figure 6 is locally knotted, globally knotted but $\pi_{1}\left(S^{3}-\tilde{\theta}\right)$ is the free group of rank 2.

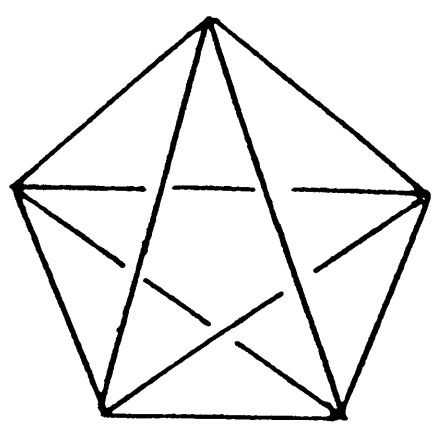

Fig. 4.

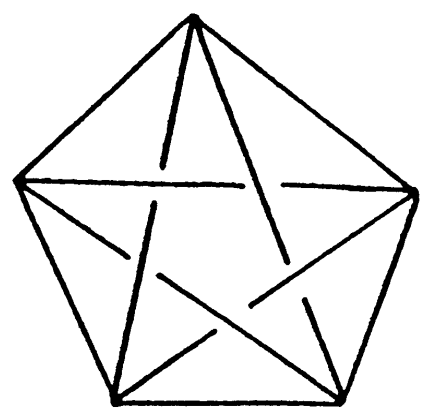

Fig. 5.

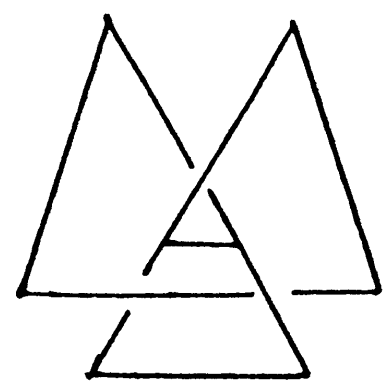

Fig. 6 . 
EXAMPLE 3. For the following spatial $\widetilde{K}_{4}$ (Figure 7 ), there are 3 cycles $x_{1}=e_{1} \cup_{\partial} e_{2} \cup_{\partial} e_{3}, x_{2}=e_{2} \cup_{\partial} e_{4} \cup_{\partial} e_{5}, x_{3}=e_{3} \cup_{\partial} e_{4} \cup_{\partial} e_{5} \cup_{\partial} e_{1}$ those which bound 2-cell respectively satisfying the conditions of Definition 1 except 2). But the set $\left\{x_{1}, x_{2}, x_{3}\right\}$ is not a base of $H_{1}\left(K_{4}: Z\right)$.

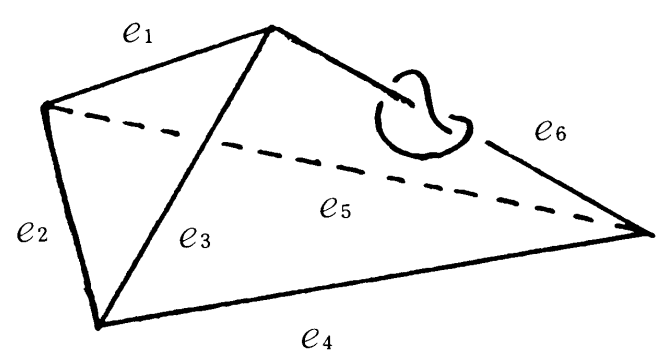

Fig. 7.

So we shall define more restricted "locally unknotted".

LEMMA 3. Let $G$ be a connected graph and $T_{G}$ be a maximal tree of G. If $E(G)-E\left(T_{G}\right)=\left\{e_{1}, \ldots, e_{\gamma}\right\}\left(\gamma=\operatorname{rank} H_{1}(G: Z)\right)$, there is a base $\left\{x_{1}, \ldots, x_{\gamma}\right\}$ of $H_{1}(G: Z)$ such that

(1) the representative curve $C_{i}$ of $x_{i}$ passes through $e_{i}$ for once.

(2) Int $\left(e_{i}\right) \cap C_{j}=\phi(i \neq j)$

PROOF. Let $\partial e_{i}=v_{i 1} \cup v_{i 2}$. Since $T_{G}$ is a maximal tree of $G$, we can take a simple path $f_{i}$ joining $v_{i 1}$ and $v_{i 2}$ in $T_{G}$. Set $C_{i}=e_{i} \cup_{\partial} f_{i}$ then $\left\{C_{1}, \ldots, C_{\gamma}\right\}$ is the set of representative curves of the required base $\left\{x_{1}, \ldots\right.$, $\left.x_{\gamma}\right\}$ by lemma 2 .

DEFINITION 6. We call the base obtained by Lemma 3 a well-situated base of $H_{1}(G: Z)$ for a maximal tree $T_{G}$. This base, of course, depends on the maximal tree $T_{G}$.

Accoding to Proposition 8 and Proposition 9, we can show the following proposition. (For the difinition of psuedo Hamiltonian, see §2.)

PROPOSITION 5. Any pseudo Hamiltonian $G$ has a spatial graph which is a locally unkotted with respect to the well situated base $\left\{x_{1}, \ldots\right.$, $x_{\gamma}$ \} for a maximal tree $T_{G}$ of $G$.

Proof. See Proposition 9.

Although the following Proposition 6 is contained in Proposition 4, the proof is more elementary. So we leave the following. 
PROPOSITION 6. If a spatial graph $\widetilde{G}$ is locally unknotted with respect to the well situated base $\left\{x_{1}, \ldots, x_{\gamma}\right\}$ for a maximal tree $T_{G}$ of $G$, then $\pi_{1}\left(S^{3}-\widetilde{G}\right)$ is a free group of $\operatorname{rank} \gamma\left(=\operatorname{rank} H_{1}(G: Z)\right)$.

PROOF. Since $G$ is locally unknotted with respect to $\left\{x_{1}, \ldots, x_{\gamma}\right\}$, there is a map $\psi: \bigcup_{i=1}^{r} D_{i}{ }^{2} \longrightarrow S^{3}$ such that

(1) $\psi\left(\partial D_{i}\right)=\widetilde{C}_{i}$

(2) $\left(\bigcup_{i=1}^{\gamma} \partial D_{i}^{2}\right)=\widetilde{G}$

(3) $\psi \mid D_{i}{ }^{2}=$ embedding

(4) $\quad \phi\left(\operatorname{Int} D_{i}^{2}\right) \cap \psi\left(\operatorname{Int} D_{j}^{2}\right)=\phi(i \neq j)$

(5) $\psi\left(D_{i}{ }^{2}\right) \cap \widetilde{G}=\psi\left(\partial D_{i}{ }^{2}\right) \cap \widetilde{G}=\widetilde{C}_{i}$ where $\widetilde{C}_{i}$ is a representative curve of $x_{i}$ on $\widetilde{G}$.

And since $\left\{x_{1}, \ldots, x_{\gamma}\right\}$ is a well-situated base for $T_{G}$, there is a set of edges, $\left\{e_{1}, \ldots ., e_{\gamma}\right\}$, such that

(6) $\left\{e_{1}, \ldots, e_{\gamma}\right\}=E(G)-E\left(T_{G}\right)$,

(7) only $\widetilde{C}_{i}$ contains $\tilde{e}_{i}$ where $\tilde{e}_{i}$ is the image of $e_{i}$ by $f$ and

(8) $\widetilde{C}_{i}$ passes through $\tilde{e}_{i}$ for once.

Since $T_{G}$ is a tree, a vertex $x_{0} \in T_{G}$ is a deformation retract of $T_{G}$ in $S^{3}$. And since each $\tilde{e}_{i}$ is a free face of a 2-complex $\widetilde{G} \cup \bigcup_{i=1}^{\gamma} \psi\left(D_{i}{ }^{2}\right)$ according to this deformation retraction, $\widetilde{G}$ deforms to a bouquet $B=\tilde{e}_{1}{ }^{\vee} \ldots \vee v \tilde{e}_{r}$ based at $x_{0}$. Each $\tilde{e}_{i}$ bounds a image $\phi\left(D_{i}{ }^{2}\right)$. And $\phi\left(\operatorname{Int} D_{i}{ }^{2}\right) \cap \phi\left(\operatorname{Int} D_{j}{ }^{2}\right)=\phi$. So $B$ is the standard bouquet in $S^{3}$. Therefore $\pi_{1}\left(S^{3}-\widetilde{G}\right)=\pi_{1}\left(S^{3}-B\right)$ and it is the free group of rank $\gamma$.

EXAMPLE. There is a spatial graph, $\widetilde{K}_{5}$, which is a locally unknotted with respect to a well situated base for a maximal tree $\Delta$ but not globally unknotted. The spatial graph $\widetilde{K}_{5}$ of Figure 8 contains a trefoil knot.

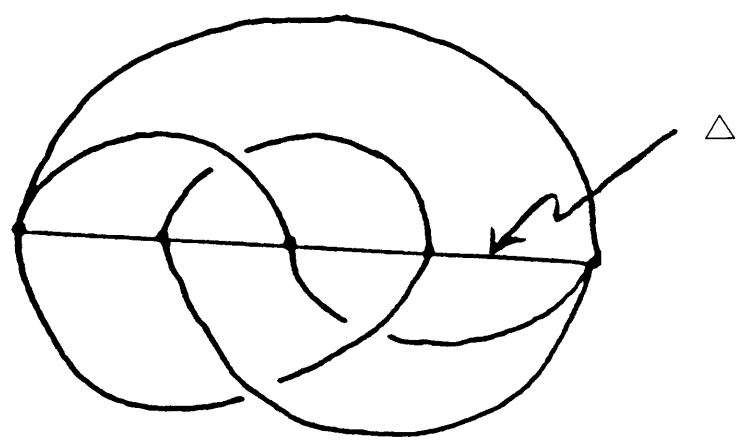

Fig. 8. 
The following definition is another restricted locally unknottedness for a comlete graph. We think that it is useful for a standardness of spatial graph of complete graphs.

DEFINITION 7. A base $\left\{x_{1}, x_{2}, \ldots, x_{\gamma}\right\}$ of $H_{1}\left(K_{n}: Z\right)$ is called a triangle base if each element $x_{i}$ of the base is represented by cycle whose length is three i.e. $x_{i}$ is representable by a complete graph with 3 vertices.

REMARK. The spatial graph, $\widetilde{K}_{5}$, of Figure 5 is locally unknotted but not locally unknotted with respect to any triangle base.

PROPOSITION 7. Any complete graph with $n$ vertices, $K_{n}$, has a spatial graph which is locally unknotted with respect to a triangle base.

PROOF. The spatial graph, $\widetilde{K}_{n}$, constructed in the proof of Theorem 1 is in fact locally unknotted with respect to a triangle base.

REMARK. Since $K_{6}$ is self-linked and $K_{7}$ is self-knotted by ([2]), there are spatial $\widetilde{K}_{6}, \widetilde{K}_{7}$ which are locally unknotted with respect to a triangle base but not globally unlinked and not globally unknotted by Proposition 7 .

QUESTION. Are there locally unknotted spatial $\widetilde{K}_{5}$ or $\widetilde{K}_{6}$ with respect to the triangle base of 1 -st homology groups of $\widetilde{K}_{5}, \widetilde{K}_{5}$ respectively which are globally knotted?

EXAMPLE. For a spatial graph, $\widetilde{K}_{5}$ (Figure 9.), we take a set of triangle cycles $B=\left\{x_{1}, x_{2}, x_{3}, x_{4}, x_{5}, x_{6}\right\}$ where $x_{1}=\langle 1 \quad 2 \quad 4\rangle, x_{2}=\langle 125\rangle, x_{3}=\langle 1$ $45\rangle, x_{4}=\langle 245\rangle, x_{5}=\langle 134\rangle, x_{6}=\langle 235\rangle$. Then every cycles $x_{i}$ 's bound a disjoint 2-disk $D_{i}{ }^{2}$ respectively . So $D_{1}{ }^{2} \cup_{\partial} D_{2}{ }^{2} \cup_{\partial} D_{3}{ }^{2} \cup_{\partial} D_{4}{ }^{2}$ is a 2-sphere and the vertices $1,2,4,5$ are on the $2^{-}$sphere. But the vertex 3 is not cotained in the 2-sphere. And the cycles $x_{5}$ and $x_{6}$ bound curved triangles respectively. So the spatial graph $\widetilde{K}_{5}$ (Figure 9) bounds 6 interior disjoint 2 -cells and the $\widetilde{K}_{5}$ obviously contains a non-trivial knot. But $B$ is not a base of $H_{1}\left(K_{5}: Z\right)$ 


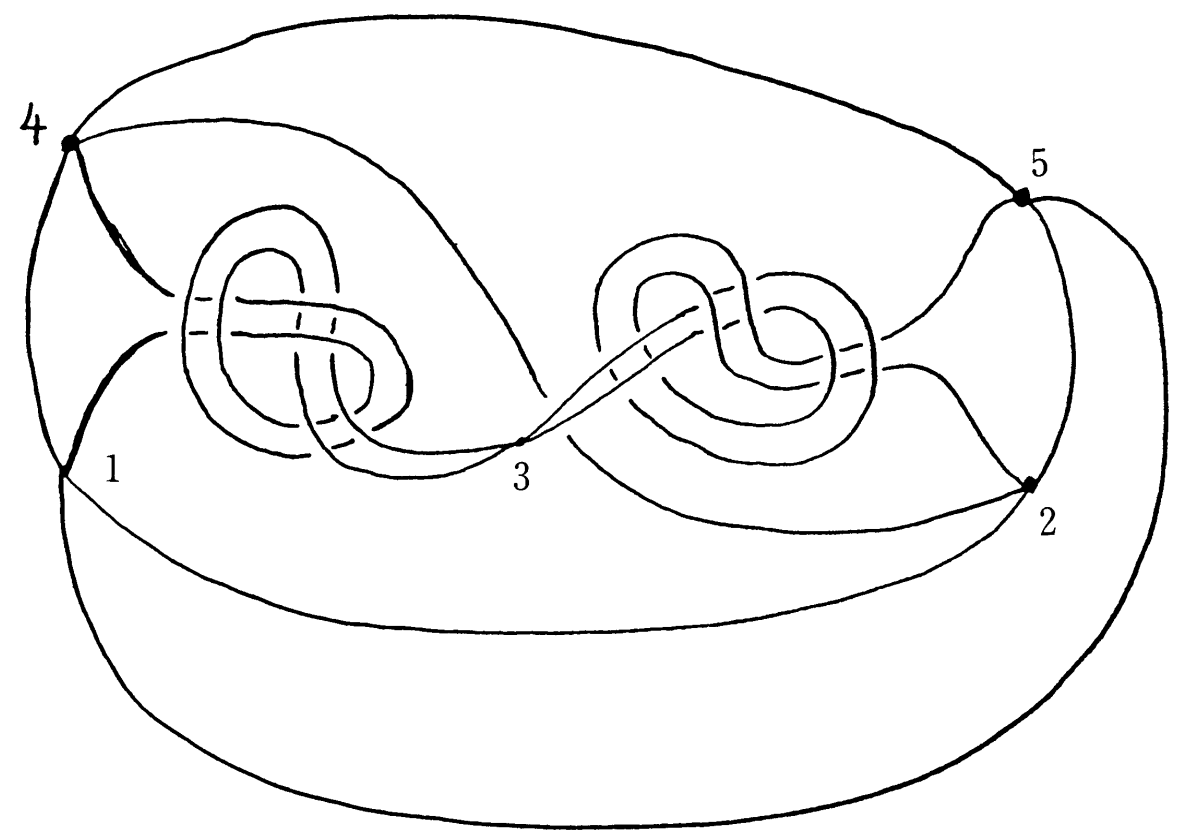

Fig. 9.

\section{§2. Book presentation.}

DEFINITION 8. Let $P_{1}^{\prime}=\left\{(x, y, z\} \in R^{3} \mid z=0, y \geqq 0\right\}$ and define inductively by

$P_{2}^{\prime}=\left\{\left(x_{2}, y_{2}, z_{2}\right) \in R^{3} \mid x_{2}=x, y_{2}=y \cos \theta-z \sin \theta, z_{2}=y \sin \theta+z \cos \theta\right.$,

$(x, y, z) \in P_{1}^{\prime}$ and $\left.\left.\theta=2 \pi / n\right\},\right\}$

$P_{k}^{\prime}=\left\{\left(x_{k}, y_{k}, z_{k}\right) \in \mid x_{k}=x, y_{k}=y \cos \theta-z \sin \theta, z_{k}=y \sin \theta+z \cos \theta\right.$,

$(x, y, z) \in P_{1}^{\prime}$, and $\left.\theta=2(k-1) \pi / \mathrm{n}\right\}$

$P_{n}^{\prime}=\left\{\left(x_{n}, y_{n}, z_{n}\right) \in R^{3} \mid x_{n}=x, y_{n}=y \cos \theta-z \sin \theta, z_{n}=y \sin \theta+z \cos \theta\right.$,

$(x, y, z) \in P_{1}^{\prime}$ and $\left.\theta=2(n-1) \pi / n\right\}$

We call $\mathscr{B}_{n}=\bigcup_{i=1}^{n} P_{i}^{\prime}$ a book. Let $\Xi=\left\{(x, y, z) \in R^{3} \mid y=z=0\right\}$ and call it the binder of $\mathscr{B}_{n}$. And let $P_{i}=P_{i}^{\prime}-\Xi$ and call it the $i$-th sheet of $\mathscr{B}_{n}$. So we can also call $\mathscr{B}_{n}=\bigcup_{i=1}^{n} P_{i}=\Xi \cup \bigcup_{i=1}^{n} P_{i}$ a book with $n$-sheets $\left\{P_{i}\right\}$ and the binder $\Xi$.

Let $\psi: G \longrightarrow \mathscr{B} n$ be an embedding satisfying that

(1) $\psi(V(G)) \subset \Xi \subset \mathscr{B}_{n}$,

(2) for any edge $e \in E(G), \phi(e) \subset \Xi$ or $\psi(\operatorname{Int}(e)) \subset P_{i}$ for some $P_{i}$ and

(3) for any sheet $P_{i}$ there is at least one edge $e$ of $G$ with $\phi(\operatorname{Int}(e))$ $\subset P_{i}$.

Then we call $\widetilde{G}=\psi(G)$ (or the embedding $\psi$ ) a book presentation of $G$ with $n$ sheets. It is clear that $0 \leqq n \leqq|E(G)|$. When $n$ is minimum, we 
call $\widetilde{G}$ a book presentation of $G$ with minimum sheets.

PROPOSITION 8. Any finite graph $G$ has a book presentation.

PROOF. We set all vertices on $\Xi$. And join the vertices by edges which are on different sheets for different edges. Then any finite graph can be embedded in a book, i. e. any finite graph has a book presentation.

DEFINITION 9. For a finite graph $G$ if there is a simple path (or simple arc) containing all vertices of $G$ we call the path a Hamilton path and $G$ a pseudo Hamiltonian. Let $\Delta$ be a Hamilton path. If a book presentation $\psi: G \longrightarrow \mathscr{B}_{n}$ satisfies a condition $\psi(\Delta) \subset \Xi$, we call $\widetilde{G}=\psi(G)$ (or 4) a book presentation with respect to a Hamilton path $\Delta$ and denote $\psi$ a B. P. H. . Furthermore if there is a simple closed path on $G$ containing all vertices of $G$ we call it a Hamilton cycle and $G$ a Hamiltonian (or a Hamilton graph).

By the same way to the proof of Proposition 8, we can prove the following.

Proposition 8'. Any pseudo Hamiltonian graph has a B. P. H. $\Delta$.

DEFINITION 10. Let $G$ be a pseudo Hamiltonian and $\Delta$ be a Hamilton path. Let $\psi: G \longrightarrow \mathscr{B} n$ be a book presentation with respect to a Hamilton path $\Delta$. If $H$ is a subgraph of $G$ containing the path $\Delta$ then we call $\widetilde{H}=$ $\psi(H)$ a spatial subgraph for the B. P. H. $\Delta$ of $G$.

PROPOSITION 9. Let $G$ be a pseudo Hamiltonian with a Hamilton path $\Delta$ and $\psi: G \longrightarrow \mathscr{B}$ p be a B.P.H. $\Delta$. Then for any spatial subgraph $\widetilde{H}$ for the B.P.H. $\Delta$ of $G, \widetilde{H}$ is locally unknotted with respect to the well-situated base $\left\{x_{1}, x_{2}, \ldots, x_{\delta}\right\}$, for a maximal tree $\Delta$ of $H$ where $\delta$ is the rank of $H_{1}(H: Z)$.

PROOF. Take a subgraph $H$ of $G$ with $H \supset \Delta$. Let $E(H)-E(\Delta)=$ $\left\{e_{1}, e_{2}, \ldots, e_{\delta}\right\}$ and $\widetilde{C}_{i}=\tilde{e}_{i} \cup_{\partial} \widetilde{f}_{i}$ be a presentation curve of $x_{i}$ on $\phi(H)$ where $f_{i}$ is a simple path on $\Delta$. Then $\widetilde{C}_{i}$ bounds a 2-disk $\widetilde{D}_{i}$ in a sheet which contains $\tilde{e}_{i}$. By deforming these 2 -disks $\left\{\widetilde{D}_{i}(i=1,2, \ldots, \delta)\right.$ a little if necessary, we can arrange $\left\{\widetilde{D}_{i}\right\}$ so that $\widetilde{D}_{i}\left(=\psi\left(D_{i}\right)\right.$ in Definition 1) satisfies the condition (1)-(5) of Definition 1. Obviously $\left\{x_{1}, \ldots, x_{\delta}\right\}$ is a well situated base of $H$ for a maximal tree $\Delta$.

COROllary 1. If $G$ is a pseudo Hamiltonian and $\psi: G \longrightarrow \mathscr{B}$ is a B. P. H. $\Delta$ then any spatial subgraph, $\widetilde{H}$, for the B. P. H. $\Delta$ of $G$ is locally unkotted. 
COROllary 2. If $G$ is a pseudo Hamiltonian and $\psi: G \longrightarrow \mathscr{B}$ is a B. P. H. $\Delta$, then for any spatial subgraph $\widetilde{H}$ for the B. P. H. $\Delta$ of $G \pi_{1}\left(S^{3}-\right.$ $\widetilde{H})$ is the free group of rank equal to $\operatorname{rank} H_{1}(H: Z)$. (This is a version of Scharleman-Thompson's Theorem ([7])).

PROOF. Since $\widetilde{H}$ is locally unknotted by Corollary $1, \pi_{1}\left(S^{3}-\widetilde{H}\right)$ is free by Proposition 4.

REMARK. If $\widetilde{H}$ is not a spatial subgraph for the B.P.H. $\Delta$ of $G$ in Corollary 2, $\pi_{1}\left(S^{3}-\widetilde{H}\right)$ is not necessarily free. For example, a spatial graph, $\widetilde{K}_{7}$, of Figure 10 contains a trefoil knot which is a spatial Hamiltonian subgraph $\widetilde{H}$ of $\widetilde{K}_{7}$ but not a spatial subgraph for the B.P.H. $\Delta$ of $K_{7}$. Also see Figure 11. A spatial graph, $\widetilde{K}_{5}$, contains a trefoil knot which is a spatial Hamiltonian subgraph $\widetilde{H}$ of $\widetilde{K}_{5}$ but not a spatial subgraph for the B.P.H. $\Delta$ of $K_{5}$.

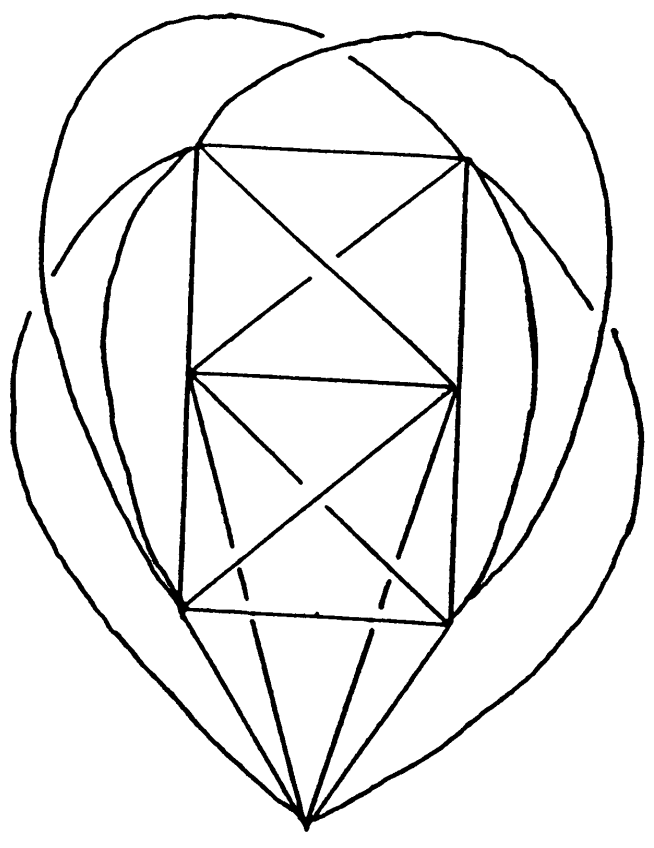

Fig. 10.
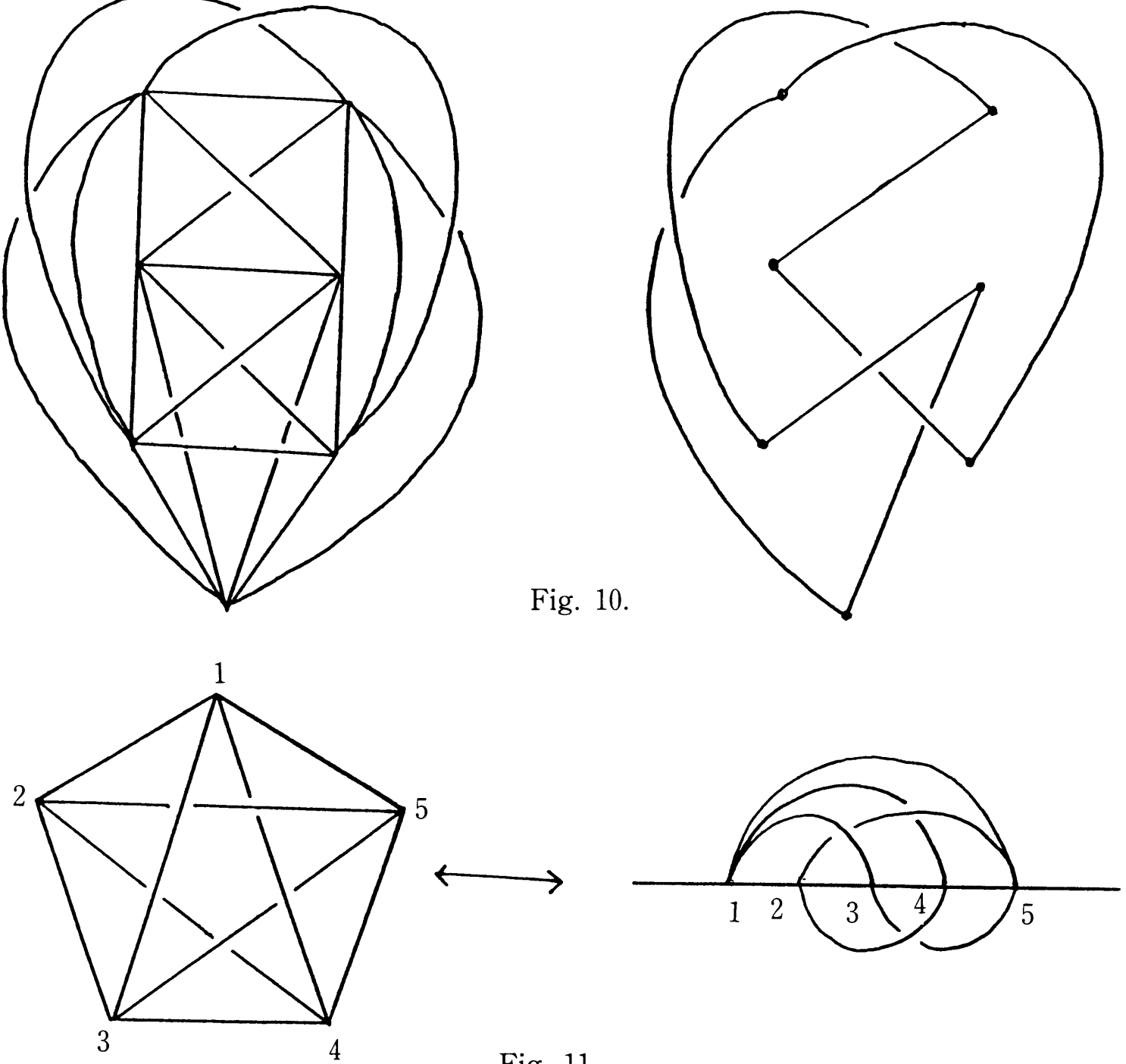

Fig. 11. 
REMARK. The converse of the above Corollary 1 in some sence does not hold, i.e. there is a locally unknotted spatial graph of a pseudo Hamiltonian which is not ambient isotopic to any B.P.H. $\Delta$ of the graph. Although the spatial graph, $\widetilde{K}_{5}$, of Figure 5 is locally unknotted, it is not ambient isotopic to a spatial graph which is a book presentation of the complete graph with 5 vertices by the following Proposition 10 because the spatial graph contains the $5_{1}$ knot. And the complete graph is a pseudo Hamiltonian (in fact it is Hamiltonian).

Combining Proposition 8' with Corollary 1 we get a following generalization of Theorem 1 .

COROLlary 3. If $G$ is a pseudo Hamiltonian, $G$ has a locally unknotted spatial graph.

Proposition 10. A. B.P. H. $\Delta$ of a complete graph $K_{5}$ can contain only trefoil knot as a non-trivial knot.

ProOF. Let $\psi: K_{5} \longrightarrow \mathscr{B} p$ be a $B . P . H$. for a Hamilton path $\Delta$. So $\psi(\Delta) \subset \Xi$. We label all vertices of $K_{5}$ via $\Delta$. Then such as Figure 12 can not occur in the book presentation. So the edges $v_{1} v_{2}, v_{2} v_{3}, v_{3} v_{4}, v_{4} v_{5}$, $v_{5} v_{1}$ in $\psi\left(K_{5}\right)$ can not have any crossing. And the remaining edges are $v_{1} v_{3}, v_{1} v_{4}, v_{2} v_{4}, v_{2} v_{5}, v_{3} v_{5}$. So possible intersections are in the pairs of

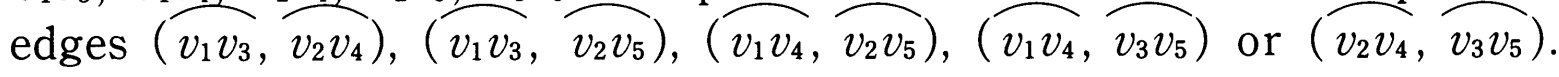
We draw these edges on the upper side of the binder. But at least 2 edges can move the lower side of the binder. So we move one edge the lower side. But every edges was used twice in the above possible intersections. So after this moving 2 intersections reduce. Hence the remaining five edges $v_{1} v_{3}, v_{1} v_{4}, v_{2} v_{4}, v_{2} v_{5}, v_{3} v_{5}$ can have only three crossings as minimal number of crossing among five sheets. So a book presentation of $K_{5}$ contains only trefoil knot as a non-trivial knot.

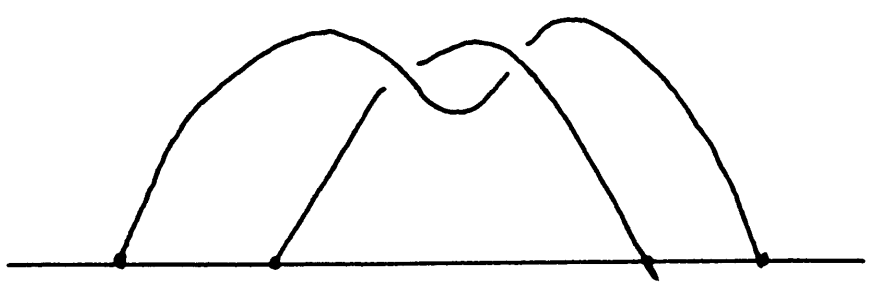

Fig. 12. 
PROBLEM. Determine the following number $c$.

A generalization of Proposition 10. A book presentation of a complete graph $K_{n}$ can contain only a knot with at most $c$ crossings as minimal crossings.

LEMMA 4. Let $\psi: K_{n} \longrightarrow \mathscr{B}_{p}$ be a B.P.H. $\Delta$ of a complete graph $K_{n}$. Then every sheets of $\mathscr{B} p$ can not contain $(n-1)$ edges.

PROOF. In this case, the maximum number of edges contained in a sheet is equal to [the number of the lines joining the opposite angle in the $n$-gon] +1 . It is $n-2$.

LEMMA 5. Let $\psi: K_{n} \longrightarrow \mathscr{B}_{p}$ be a B.P.H. $\Delta$ of a complete graph. Then there is at most one sheet containing $(n-2)$ edges.

PROOF. Every sheet can not contain $(n-1)$ edges by lemma 4 . And the edge $1 n$ can be contained every sheets. So to contain $(n-2)$ edges in a sheet we have to use the edge $1 n$. Hence there is only one sheet containing $(n-2)$ edges.

THEOREM 2. Let $K_{n}$ be the complete graph with $n$ vertices and $\psi: K_{n}$ $\longrightarrow \mathscr{B}_{p}$ be a B.P. H. $\Delta$ with $p$-sheets, then $[(n+1) / 2] \leqq p \leqq(n-1)(n-2) / 2$ for $n \geqq 4$ and $p=1$ for $n=3$ where $[k]$ is greatest integer less than or equal to $k$. $-1) / 2$.

PROOF. For $n=3$ it is obvious. And obviously $0 \leqq p \leqq\left|E\left(K_{n}\right)\right|=n(n$

Since $\psi$ is a $B . P . H . \Delta$, there are $(n-1)$ edges on the binder $E, n(n$ $-1) / 2-(n-1)=(n-1)(n-2) / 2$. Hence $p \leqq(n-1)(n-2) / 2$. And any sheet can not contain $(n-1)$ edges by lemma 4 . And there is only one sheet which contains $(n-2)$ edges by lemma 5 . So other sheets contain at most $(n-3)$ edges. Therefore $(n-1)+(n-2)+(p-1)(n-3) \geqq n(n-1) / 2$ and $p \geqq n / 2$. Since $p$ is an integer $p \geqq[(n+1) / 2]$.

If there is a B.P.H. $\Delta$ with 2 sheets, $\psi: G \longrightarrow \mathscr{B}_{2}$, then $\phi(G)$ is a plane graph. The following Proposition states the converse for Hamiltonian (not pseudo Hamilton) and determine the minimum number of sheets of the B.P.H. $\Delta$ for the complete graph.

Proposition 11. (1) Let $\widetilde{G}$ be a plane Hamiltonian. Then there is a B.P.H. $\Delta$ of $G$ with 2 sheets, $\psi: G \longrightarrow \mathscr{B}_{2}$ which is ambient isotopic to $\widetilde{G}$.

(2) [T. Endo, K. Ikeda, A. Kaneko and C. Toba] Let $K_{n}$ be a complete graph and $\psi: K_{n} \longrightarrow \mathscr{B}_{p}$ a B.P.H. $\Delta$ of $K_{n}$ with the minimum num- 
ber of sheets. Then $p=[(n+1) / 2]$.

Proof. (1) Since $\widetilde{G}$ is a plane graph, $\widetilde{G}$ is on a 2 -sphere $S^{2}$. And since $G$ is a Hamiltonian, there is a Hamilton cycle $\widetilde{C}$ on $\widetilde{G}$ which divides $S^{2}$ into two 2-balls by Scoenflies Theorem (or Jardan Curve Theorem). We isotope $\widetilde{C}$ to the equator $K$. By considerinsg the north hemishere the first sheet and the south hemisphere the second sheet, we can construct a B.P.H. $\Delta$ of $G, \psi: G \longrightarrow \mathscr{B}_{2}$ with 2 sheets whose image $\psi(\widetilde{G})$ is ambient isotopic to $\widetilde{G}$.

(2) First we prove the case $n$ even, $n=2 m$. Let $V\left(K_{2 m}\right)=\left\{v_{1}, v_{2}, \ldots\right.$, $\left.v_{2 m}\right\}$. On the $i$-th sheet $P_{i}(1 \leqq i \leqq m)$ we draw edges $v_{i} v_{i}+2, v_{i} v_{i}+3, \ldots$, $v_{i} v_{i+m}$ and $v_{i+m} v_{i+m+2}, \ldots, v_{i+} v_{i+2 m-1}$ where the number of vertices is of mod $2 m$. And draw the edges $v_{i} v_{i+1}, v_{i+m} v_{i+m+1}$ on the binder $\Xi$. By the contruction, we get a B.P.H. $\Delta$ of $K_{2 m}, \psi: K_{2 m} \longrightarrow \mathscr{B}_{m}$.

The case $n$ odd, $n=2 m+1$. Let $V\left(K_{2 m+1}\right)=\left\{v_{1}, v_{2}, \ldots, v_{2 m} v_{2 m+1}\right\}$ and $V_{0}=\left\{v_{1}, \ldots, v_{2 m}\right\}$. Then we may consider $K_{2 m+1}=K_{2 m} \cup\left(V_{0} * v_{2 m+1}\right)$ where $V_{0} * v_{2 m+1}$ is a set of edges obtained by joining every vertices in $V_{0}$ with $v_{2 m+1}$. We draw $\widetilde{K}_{2 m}$ in a book $\mathscr{B}_{m}$ as a B.P.H. $\Delta$. Add one sheet $P_{m+1}$ and the last vertex $v_{2 m+1}$ on the binder $\Xi$. And by joining the vertex $v_{2 m+1}$ with the remaining vertices $v_{1}, \ldots, v_{2 m}$ on the new sheet $P_{m+1}$, we get a $B$. P.H. $\Delta \psi: K_{2 m+1} \longrightarrow \mathscr{B}_{m+1}$. These number of sheets are minimum by Theorem 2.

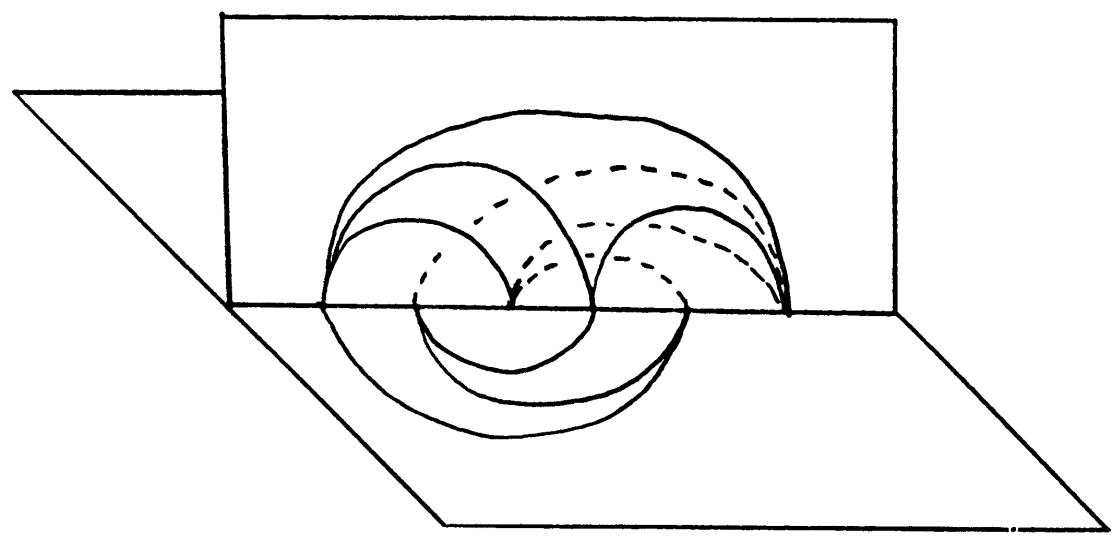

Fig. 13. 
DEFINITION 11. Let $\mathscr{B}_{p}=\Xi \cup \bigcup_{i=1}^{p} P_{i}$ be a book with $p$ theets where $P_{i}$ is an $i$-th sheet. And let $h: \mathscr{B}_{p} \longrightarrow \mathscr{B}_{p}$ is a homeomorphism such that

(1) $h \mid \Xi=i d$.

(2) $h\left(P_{i}\right)=P \sigma_{(i)}$ where $\sigma$ is a permutation of $\{1,2, \ldots, p\}$. Then we define $h$ to be a sheet translation.

PROPOSITION 12. Let $\psi^{\prime}: K_{n} \longrightarrow \mathscr{B}_{q}$ be a B. P. H. $\Delta$ with maximum $q$ sheets. Then there is a sheet translation $h: \mathscr{B}_{q} \longrightarrow \mathscr{B}_{q}$ so that $h \psi^{\prime}\left(K_{n}\right)$ is ambient isotopic to a spatial graph $\psi\left(K_{n}\right)$ where $\psi: K_{n} \longrightarrow \mathscr{B}_{p}$ is a B.P. $H . \Delta$ with minimum $p$ sheets.

PRoOF. Take a B.P.H. $\Delta \quad \psi: K_{n} \longrightarrow \mathscr{B}_{p}$ with minimum sheets. And peel every edges from sheet and set each edge in one sheet. And use the sheet translation $h$, we can deform $h \psi^{\prime}$ to $\psi$ up to an ambient isotopy.

REMARK. According to Proposition 12, if we use a finite number of times of sheet translations and ambient isotopies, then all spatial graphs, $\widetilde{K}_{n}$, of the complete graph with $n$ vertices are ambient isotopic each other. So we can use only one time of sheet translation and an ambient isotopy.

PROPOSITION 13. Let $K_{5}$ be the complete graph with 5 vertices and $\psi$ : $K_{5} \longrightarrow \mathscr{B}_{3}$ be a B,P.H. $\Delta$ with minimum sheets of $K_{5}$ then $\psi\left(K_{5}\right)$ is unique up to homeomorphism of $S^{3}$. Furthermore $\psi\left(K_{5}\right)$ is unique up to ambient isotopy of $S^{3}$. And $\psi\left(K_{5}\right)$ is globally unknotted.

PROOF. Let $h$ be a sheet translation of $\mathscr{B}_{3}$ corresponding to a permutation $\sigma=(3,2)$. Then $h$ is a homeomorphism defined by $h(x, y, z)=(x, y$, $-z)$. So $h$ is an orientation reversing homeomorphism of $R^{3}$. Since $\mathscr{B}_{3}$ has only 3 sheets, any sheet translation is an orientation preserving or reversing homeomorphism. And possible images of $K_{5}$ by a B.P.H. $\Delta$ up to a $\pi$-rotation of $\Xi$ arround the $z$-axis are the followings $1-1,1-2$, $2-1,2-2$ of Figure 14.; 

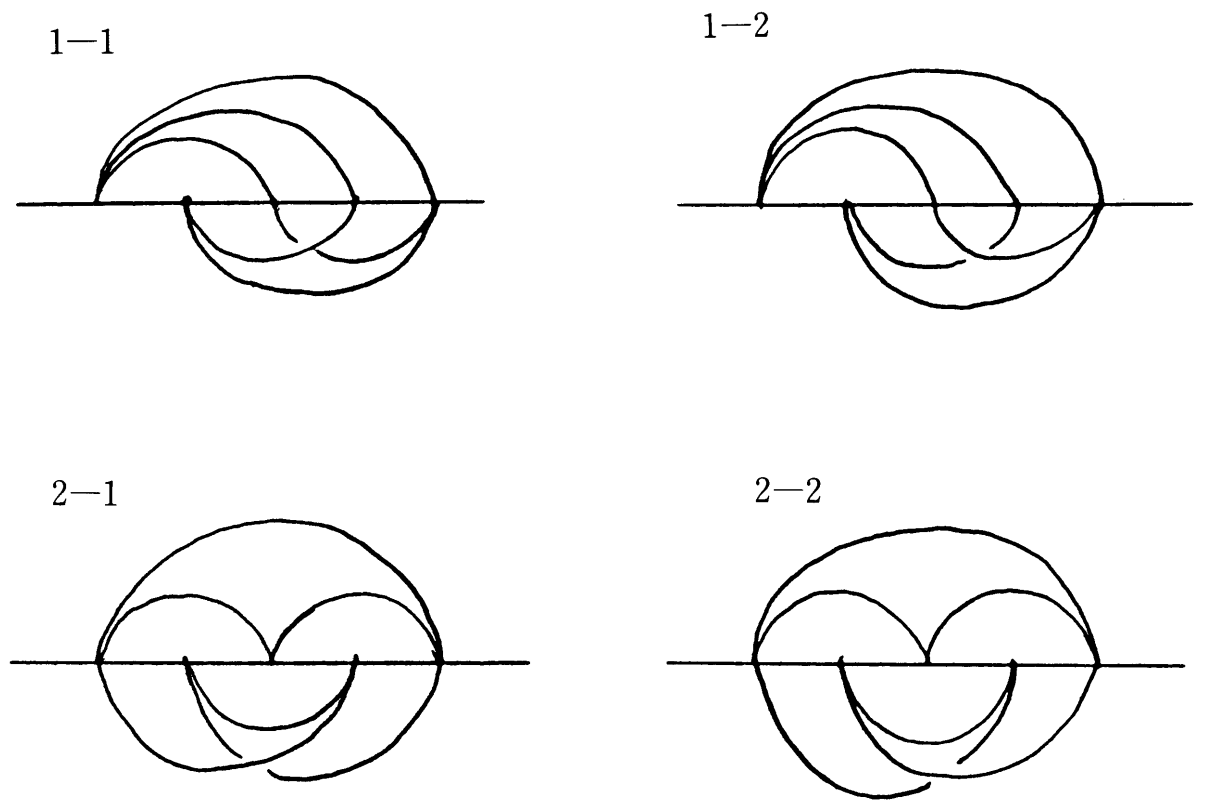

Fig. 14.
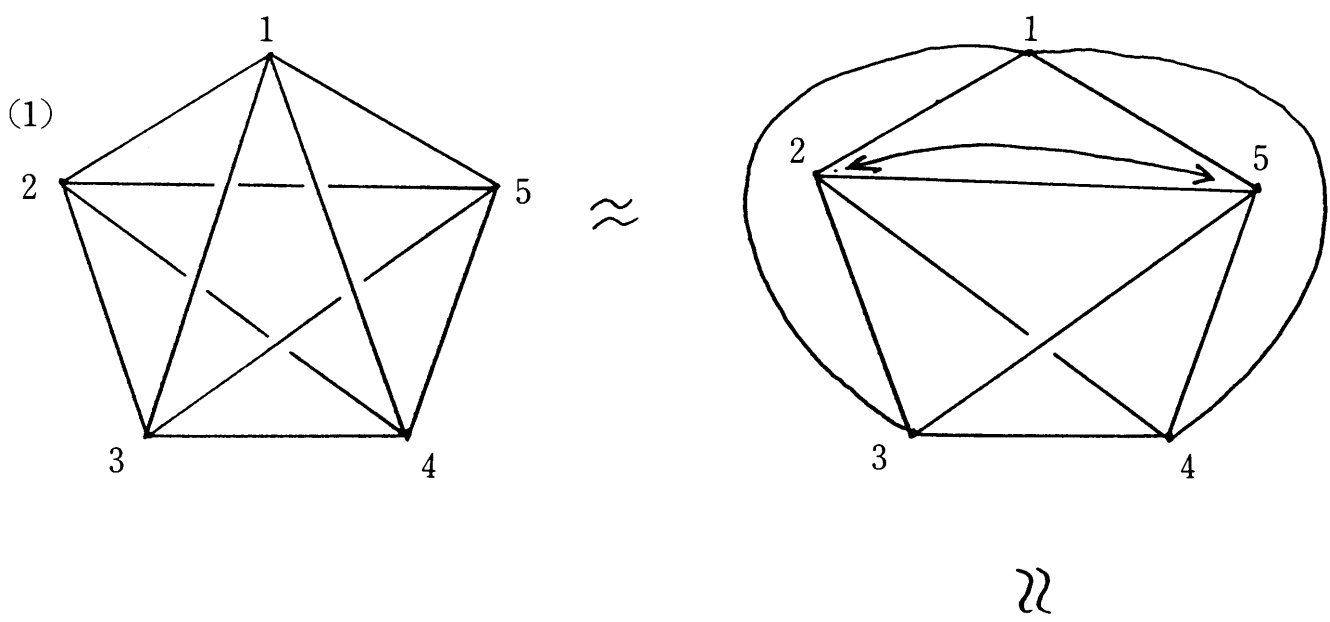

(2)
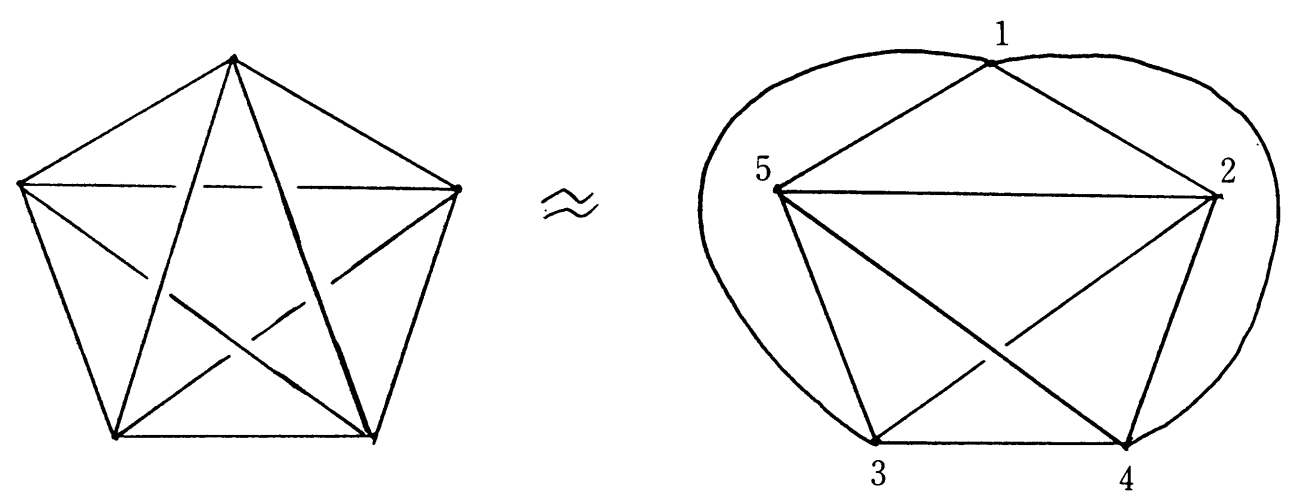

Fig. 15. 
$1-1$ (resp. 2-1) is ambient isotopic to $1-2$ (resp. 2-2) after sheet translation. $1-1$ (resp. 1-2) is ambient isotopic to $2-1$ (resp. 2-2). So $\psi\left(K_{5}\right)$ is unique up to homeomorphism of $S^{3}$. And $1-1$ (resp. 1-2) is ambient isotopic to (1) (resp. (2)) of Figure 15. Then take the edges $v_{1} v_{3}$ and $v_{1} v_{4}$ in the diagram (1) of Figure 15 outside of the pentagon and change the vertices $v_{2}$ and $v_{5}$ isotopically keeping the vertices $v_{1}, v_{3}$ and $v_{4}$ fixed. At the last take the edges $v_{1} v_{3}$ and $v_{1} v_{4}$ into the pentagon, then we get the diagram (2). Hence the diagram (1) and (2) of Figure 15 are ambient isotopic each other. So $\psi\left(K_{5}\right)$ is unique up to ambient isotopy of $S^{3}$ and it is obviously globally unknotted.

COROLlaRY. Let $\psi: K_{5} \longrightarrow \mathscr{B}_{3}$ be a B. P. H. $\Delta$ with minimum sheets. Then $\psi\left(K_{5}\right)$ is locally unknotted with respect to a triangle base.

PROOF. If $\psi$ is a B.P.H. $\Delta$ with minimum sheets, then $\psi\left(K_{5}\right)$ is one of Figure 14. And they are all locally unknotted with respect to a triangle base.

REMARK. In general we can not extend the second part of Proposition 13 to any B.P.H. $\Delta$ with minimum sheets, $\psi: K_{n} \longrightarrow \mathscr{B} p, p=[(n+1) /$ 2]. For example, $K_{7}$ has two kind of B.P.H. $\Delta$ with 4 sheets such that one is a mirror image of the other. So one contains a right handed trefoil knot and the other one contains a left handed trefoil knot (Fig. 16.). So these two spatial graphs $\widetilde{K}_{7-1}$ and $\widetilde{K}_{7-2}$ of $K_{7}$ are not ambient isotopic each other. But $\widetilde{K}_{7-1}$ changes to $\widetilde{K}_{7-2}$ by a sheet translation with $\sigma=\left(\begin{array}{ll}1 & 4\end{array}\right)$ (2 3 ). This sheet translation extends to an orientation reversing homeomorphism of $S^{3}$. 

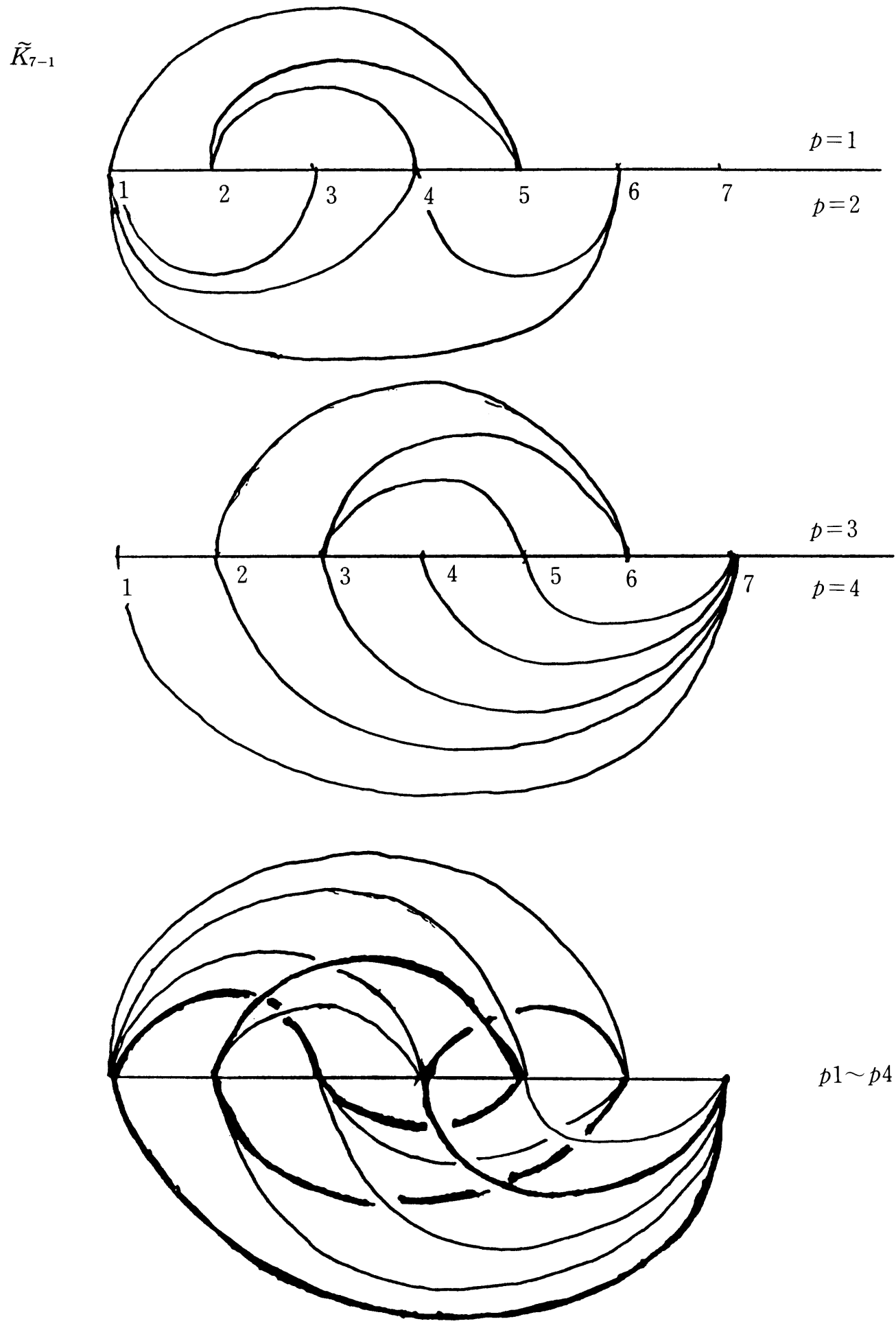

$p 1 \sim p 4$

Fig. 16-1 

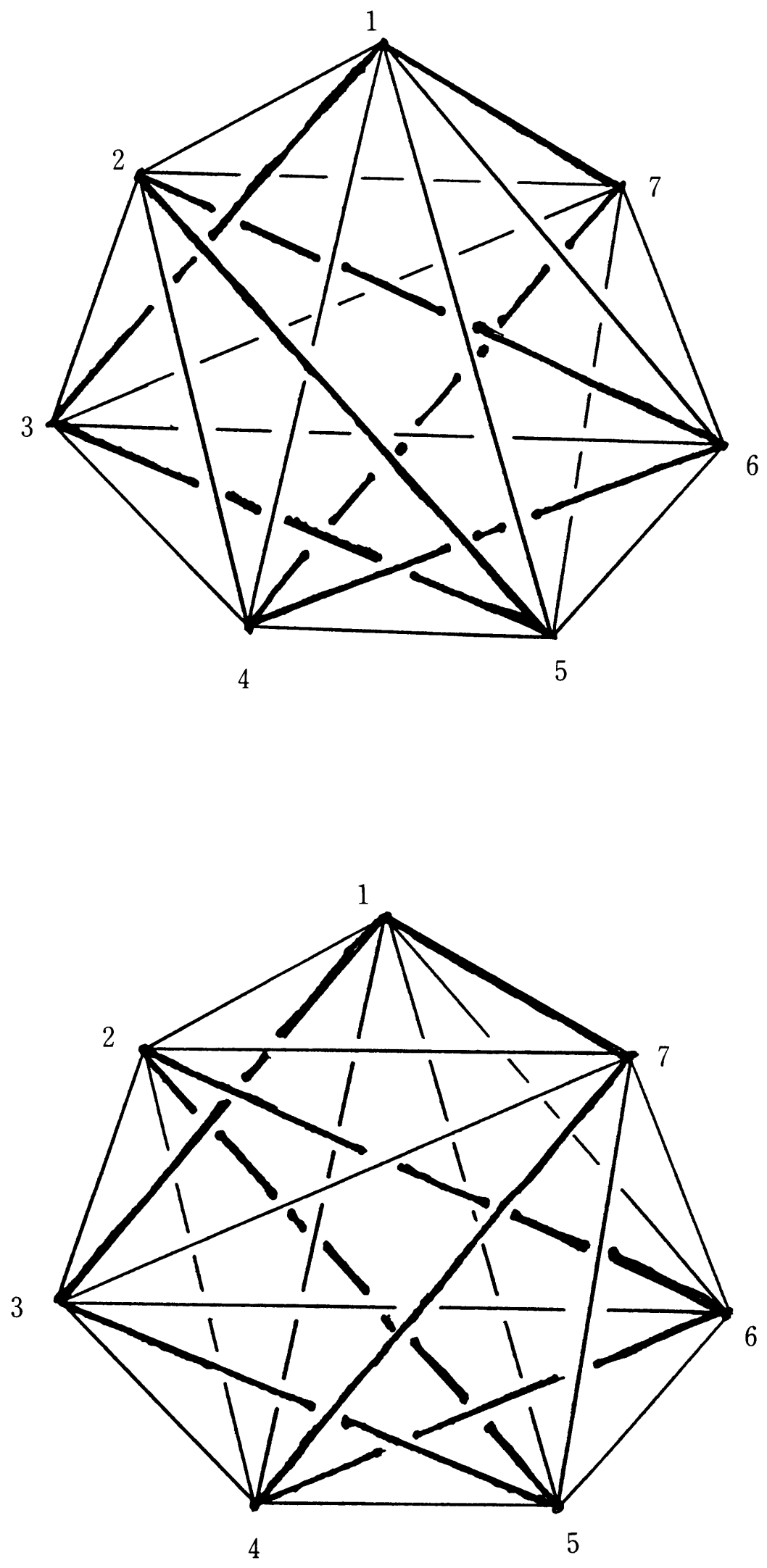

Fig. 16-2 
QUESTION. Can we generalize the first part of Proposition 13 for any comlete graph, $K_{n}$ ?

REMARK. Although for a B.P.H. $\Delta$ of $K_{6}$ with the minimum number of sheets $\psi: K_{6} \longrightarrow \mathscr{B}_{3}, \widetilde{K}_{6}=\psi\left(K_{6}\right)$ contains a Hopf link, it does not contain any non-trivial knot. Because any cycle $\widetilde{C}$ on $\widetilde{K}_{6}$ is a 2 -bridge knot for which the upper bridges is contained in the first sheet (Figure 17.). But any 2-bridge knot with 6-vertices in the standard form of Schubert is the trivial knot. So $K_{6}$ does not contain a non-trivial knot and it is globally unknotted. This method is also applicable to the proof of Proposition 13.

CONJECTURE. Any B.P.H. $\Delta$ with minimum sheets $\psi: K_{n} \longrightarrow \mathscr{B}_{p}$ is equivalent up to the sheet translation of $\mathscr{B}_{p}$ and the ambient isotopy.

At the present time, in spite of the above conjecture, we think the following definition is suitable for the standard spatial graph of the pseudo Hamiltonian.

DEFinition 12. Let $G$ be a pseudo Hamiltonian and $\psi: G \longrightarrow \mathscr{B} p$ be a B.P.H. $\Delta$ with minimum sheets. We call a spatial graph $\widetilde{G}$ of $G$ the standard spatialgraph of $G$ if $\widetilde{G}$ is ambient isotopic to the spatial graph $\psi(G)$.

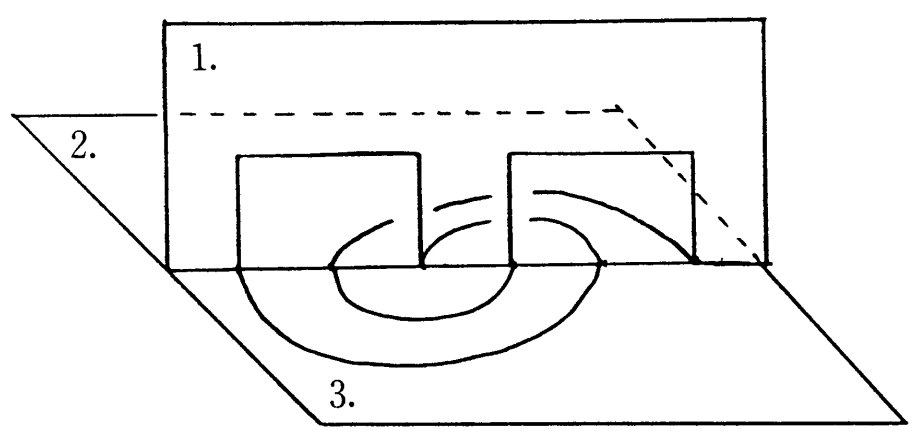




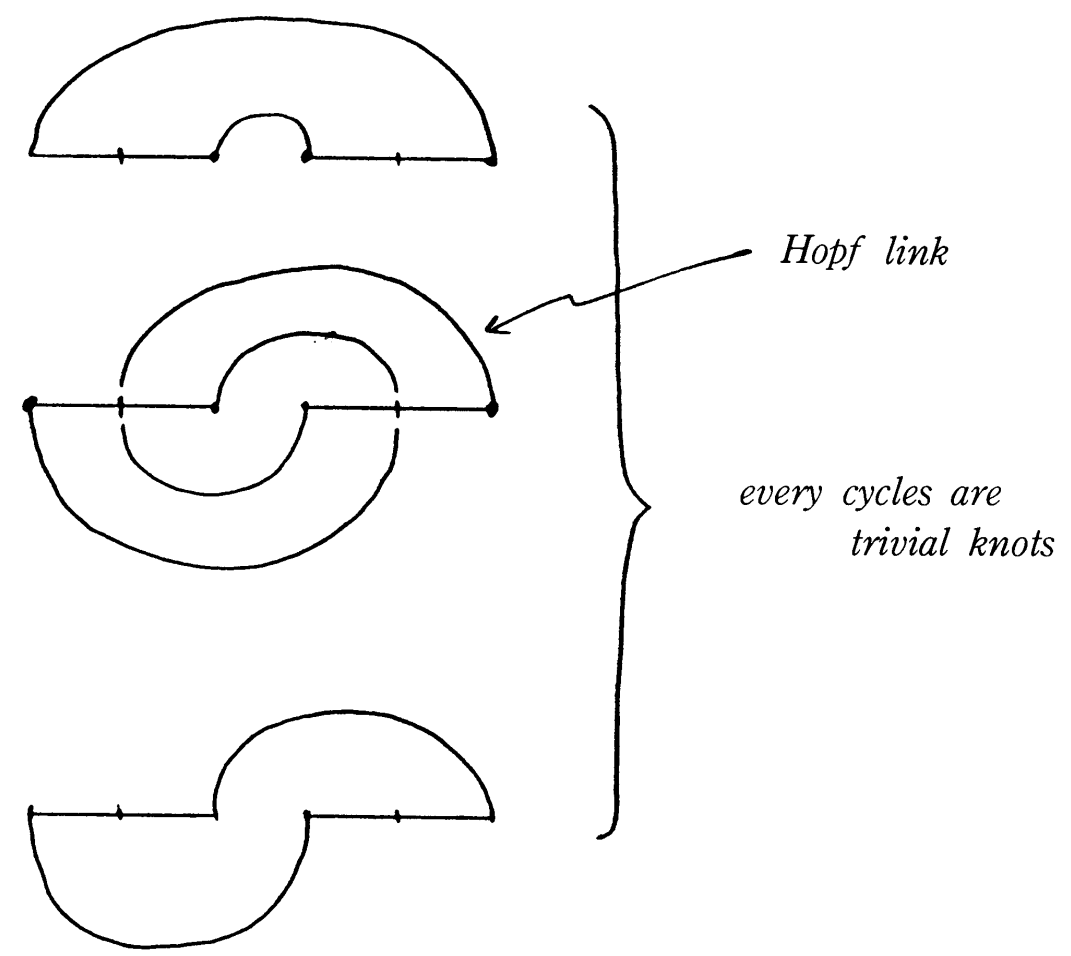

Fig. 17

\section{References}

[1] BONDY, J. A. \& MURTY, U.S.R.: Graph theory with application, North Holland, (1976) New York, Oxford.

[2 ] Conway, J. H. \& GORDON, C. McA. : Knots and links in spatial graphs, J. Graph thoery, 7 (1985) 445-453

[ 3 ] Hempel, J. : 3-manifolds, Ann. of Math Studies 86, (1976) Princeton Univ. Press.

[4] IKEDA, K.: On minimum sheet numbers of graphs, (preprint)

[5] KOHARA, T. \& SUZUKI, S. : Some remarks on knots and links in spatial graph, (preprint)

[ 6 ] PAPAKYRIAKOPOUlos, C. D.: On Dehn's lemma and asphericity of knots, Ann. of math. 66 (1975) 1-26

[ 7 ] Scharleman, M. \& Thompson, A.; Detecting unknotted graphs in 3-space, (preprint)

[ 8] SHImabarA, M. : Knots in certain spatial graphs, Tokyo J. Math. 11 (1988) 405-413

[ 9] SUZUKI, S.: Constituent knots and links of spatial graphs, RIMS kokyuroku 686 (1989) "Topological Graph Theory" Kyoto Univ. 55-86

[10] : Knots and links in spatial graphs II, (in Japanese) Gakujutu Kenkyu, School of Education, Waseda Univ. Series of Math. 38 (1989) 21-28.

[11] White, A. T.: Graphs, Groups and Surfaces, Mathematics Studies 8 Revised edition, (1984) North Holland, New York.

Dapartment of Mathematics

Faculty of Literature and Science

Tokyo Woman's Christian University

Tokyo, 167 Japan 